\title{
Equipo comparativo para la medida de conductividad térmica de materiales cerámicos
}

\author{
'R. BAREA, ${ }^{2}$ R. MARÍN, ${ }^{1}$ M.I. OSENDI, ' $R$. MARTINEZ Y 'P. MIRANZO. \\ 'Instituto de Cerámica y Vidrio, CSIC. Cantoblanco. Madrid.. \\ ${ }^{2}$ Ikerlan Energía, Centro de Investigaciones Tecnológicas, Parque Tecnológico de Álava, Miñano Menor, Álava.
}

\begin{abstract}
Entre las distintas técnicas de medida de la conductividad térmica de materiales, el método comparativo, recogido en la norma ASTM E1225-99, es el más adecuado para muestras porosas o materiales compuestos. Este método permite determinar conductividades térmicas en estado estacionario, en el intervalo de valores de 0,2 a $200 \mathrm{~W} / \mathrm{mK}$, y a temperaturas de ensayo en el rango 100-1300 K, dependiendo del diseño del equipo. En este trabajo se expone el diseño, desarrollo y puesta a punto de un equipo de medida de conductividad térmica basado en este método. Paralelamente, se realiza una validación del equipo mediante el estudio comparativo de los resultados obtenidos usando el equipo comparativo y la técnica del pulso láser en materiales porosos de mullita.
\end{abstract}

Palabras clave: conductividad térmica, difusividad térmica, método comparativo, materiales porosos, mullita.

Comparative equipment for the measurement of the thermal conductivity.

Among the different techniques available for measuring thermal conductivity, the comparative method (ASTM E1225-99) is the most appropriate for porous materials. This method determines the thermal conductivity in steady state conditions in the 100 - 1300 K temperature range and for conductivities between 0.2 and $200 \mathrm{~W} / \mathrm{mK}$, respectively, depending on the equipment characteristics. In the present paper, the design and development, as well as the start up process, of an apparatus based on this method is described. Thermal conductivity data on a mullite based refractory, measured by both the laser flash and the comparative methods, are presented and comparatively discussed in order to validate the developed equipment.

Keywords: thermal conductivity, thermal diffusivity, comparative method, porous materials, mullite.

\section{INTRODUCCIÓN}

Desde el punto de vista socioeconómico, el estudio de los procesos de conducción de calor en los materiales cerámicos, así como la determinación experimental precisa de la conductividad térmica, son temas de una gran importancia para muchos sectores industriales y de consumo. Entre aquellos se pueden citar la industria tradicional cerámica, la de construcción, la industria metalúrgica, y la industria del electrodoméstico. Además, se puede afirmar que la conductividad térmica es un parámetro clave en el diseño y desarrollo de nuevos materiales y recubrimientos para las industrias aerospacial, de automoción y de generación de energía.

La determinación de la conductividad térmica es compleja. Generalmente, se requieren distintos equipos de medida para abarcar los amplios rangos de conductividades, de temperaturas y de tipos de materiales (Figura 1). De hecho, en la literatura científica se pueden encontrar numerosos trabajos dedicados exclusivamente al estudio comparativo de los diversos métodos de medida $(1,2)$. La mayoría de los equipos están diseñados para piezas de gran tamaño $(23 \times 11,4 \times 6,4$ $\left.\mathrm{cm}^{3}\right)$, por lo que su utilización queda restringida a mantas de fibras aislantes y refractarios. En el otro extremo, la técnica de pulso láser permite determinar la difusividad térmica en un amplio intervalo de difusividades, entre $10^{-7}$ y $10^{-5} \mathrm{~m}^{2} / \mathrm{s}, \mathrm{y}$ temperaturas, de 100 a $2300 \mathrm{~K}$, pero en muestras de tamaño muy pequeño, $12 \mathrm{~mm}$ de diámetro y 1-3 $\mathrm{mm}$ de espesor (3). Esto, que en principio es una ventaja en el caso de los materiales avanzados, generalmente muy homogéneos y de tamaños de grano y de poro pequeños, es una limitación cuando se trata con materiales de características microestructurales en el rango del milímetro. Además, esta técnica no es recomendable para muestras con altas porosidades porque el láser puede traspasar directamente la muestra si los poros son grandes, $y$, además, se comete un error en la medida del espesor real de las muestras, debido a la rugosidad superficial asociada a la porosidad (4-6).

El objetivo fundamental de este trabajo ha sido el diseño y desarrollo de un equipo para la medida de la conductividad térmica capaz de trabajar en un amplio intervalo de temperaturas y conductividades, y que se pueda utilizar para diferentes tipos de materiales, complementario del equipo de medida de la difusividad por la técnica del pulso-láser. Como base para el diseño del nuevo equipo, se ha optado por el método comparativo (7-10) ya que permite obtener resultados fiables en un intervalo amplio de conductividades, entre 0,2 y $200 \mathrm{~W} / \mathrm{mK}$, y hasta temperaturas de $1300 \mathrm{~K}$. Estos intervalos pueden ampliarse a costa de perder en precisión. La medida de la conductividad se realiza en régimen estacionario y emplea un material de conductividad térmica conocida como patrón para medir el flujo de calor. Las muestras son de tamaño medio ( $50 \mathrm{~mm}$ de diámetro y $25 \mathrm{~mm}$ de altura) pero suficientemente representativas del material en el caso de microestructuras en el rango del milímetro. 


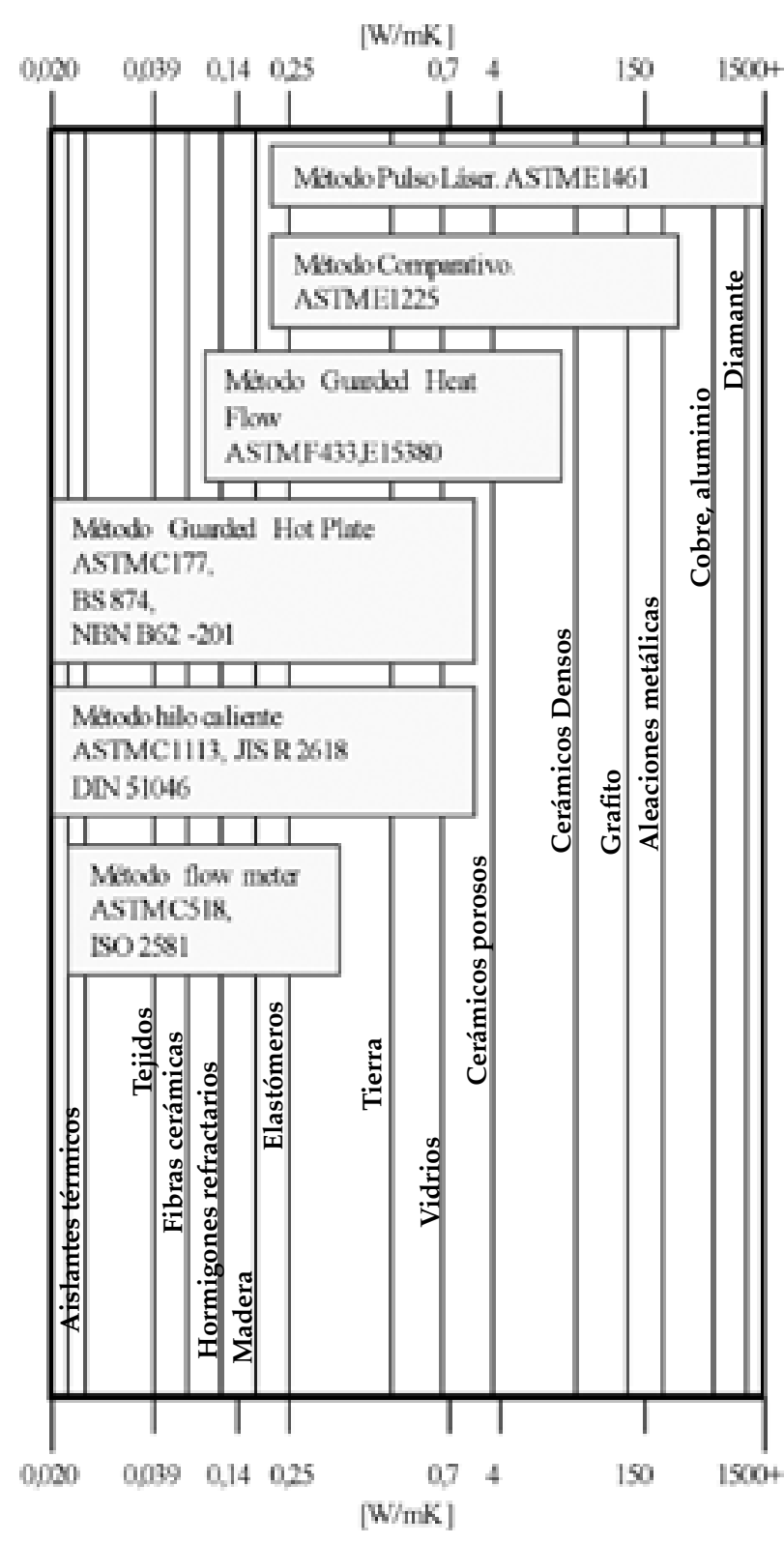

Fig. 1.- Esquema de los diferentes métodos de medida de conductividad térmica, representados frente al intervalo de aplicación de cada uno de ellos, en el que se ha indicado la correspondiente normativa y el rango de conductividades de algunos materiales.

\section{PRINCIPIO DEL MÉTODO COMPARATIVO}

El método comparativo se denomina así porque esta basado en la comparación entre los gradientes térmicos que se establecen entre dos probetas patrón y la muestra problema situada entre ambas. El diseño del equipo se ha abordado considerando la solución de la ecuación del calor en régimen estacionario para el caso de un flujo de calor unidimensional, sin sumideros ni fuentes de calor. La configuración experimental más empleada consiste en un cilindro en el que se impone un gradiente de temperaturas entre los dos extremos. La resolución de la ecuación del calor se aborda suponiendo las siguientes condiciones de contorno (c.c.): temperatura fija y constante en dos puntos separados una distancia $\mathrm{L}$, igual a la longitud del cilindro, y pérdidas de calor nulas en cualquier dirección distinta a la de propagación (z). Esto es:

$$
\begin{gathered}
d T / d r=\mathrm{C} \\
T(0)=T_{1} \\
T(L)=T_{-}
\end{gathered}
$$

Con estas tres condiciones de contorno, se obtiene el siguiente perfil de temperaturas:

$$
T(z)=\frac{\left(T_{2}-T_{1}\right)}{L} z+T_{1}
$$

A partir de la solución anterior y empleando la ecuación de Fourier, que relaciona el flujo de energía térmica, $\mathrm{Q}$, a través de un área, A, con el gradiente de temperatura, se obtiene la conductividad térmica, $\mathrm{k}$, del material:

$k=-\frac{Q}{A} \frac{L}{\left(T_{2}-T_{1}\right)}$

Basándose en esta solución, para realizar la medida con esta técnica, la muestra se coloca entre dos patrones de conductancia térmica (conductividad térmica/espesor) igual o superior a la muestra problema, formando lo que se denomina el apilamiento de medida (Figura 2). A continuación, se establece un gradiente térmico entre la parte superior $\left(\mathrm{T}_{1}\right)$ e inferior $\left(\mathrm{T}_{6}\right)$ del apilamiento de medida, esto es $\Delta \mathrm{T}=\mathrm{T}_{1}-\mathrm{T}_{6^{\prime}}$ y se aseguran las condiciones adiabáticas del sistema. Una vez alcanzado el equilibrio térmico, se mide el gradiente de temperatura final alcanzado en ambos patrones y en la muestra. Si se cumpliesen las condiciones de régimen estacionario, no existiesen resistencias térmicas de contacto en el apilamiento de medida y las áreas de contacto fuesen estrictamente iguales, podríamos asumir que el flujo térmico es el mismo en la muestra y en los patrones superior e inferior, por lo que la conductividad térmica de la muestra se puede obtener igualando el flujo de calor en la muestra al flujo en el patrón superior o en el patrón inferior. La norma ASTM E1225 (10) propone promediar el resultado de ambas igualdades para minimizar el error, lo que conduce a la siguiente expresión:

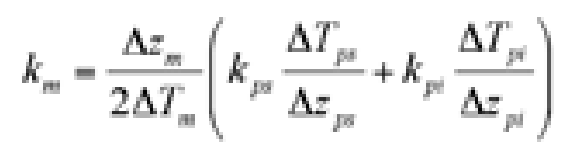

donde $\Delta z$ es la distancia entre la que se establece la diferencia de temperatura $\Delta \mathrm{T}$. Los subíndices $\mathrm{m}$, ps y pi se refieren a muestra, patrón superior y patrón inferior, respectivamente. 

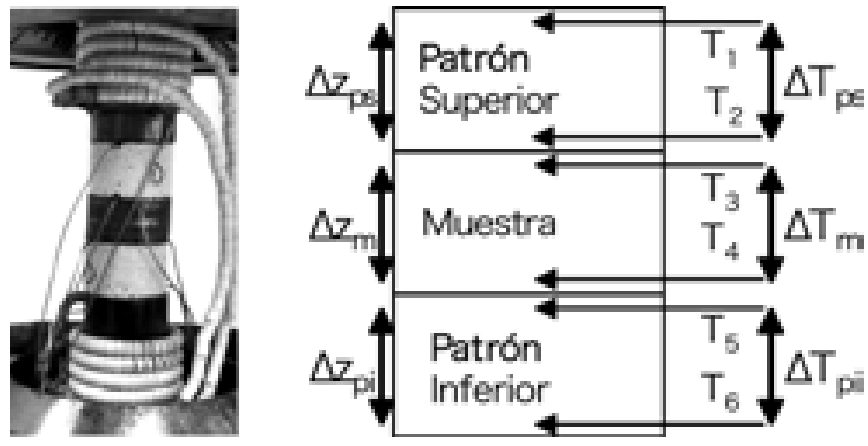

Fig. 2.- Vista del apilamiento de las muestras junto con el esquema del método de medida.

\section{ANÁLISIS DEL ERROR DE LA MEDIDA}

\subsection{Propagación de errores.}

En primer lugar, se ha realizado una estimación de la incertidumbre de la medida mediante propagación de errores (11) en la expresión [4]. Para ello, se ha supuesto que el error en todas las medidas de distancia entre termopares es el mismo e igual a $\delta(\Delta z)$, que el error en la medida del gradiente de temperatura es el mismo e igual a $\delta(\Delta \mathrm{T})$, y que los valores de conductividad térmica tienen la misma incertidumbre, $\delta \mathrm{k}_{\mathrm{p}}$. Con ello, se llega a la siguiente expresión:

$$
\begin{aligned}
& \left(\frac{\delta k_{m}}{k_{m}}\right)^{2}=\left(\frac{k_{n} \delta \Delta z}{\Delta z_{m}}\right)^{2}+\left(\frac{k_{m} \delta \Delta T}{\Delta T_{m}}\right)^{2}+\left(Q_{m}^{2}+Q_{p}^{2}\right)\left(\frac{\delta k_{p}}{k_{p}}\right)^{2} \\
& +\left(\frac{Q_{m}^{2}}{\Delta T_{m}^{2}}+\frac{Q_{m}^{2}}{\Delta T_{m}^{2}}\right) \delta \Delta T^{2}+\left(\frac{Q_{p}^{2}}{\Delta z_{m}^{2}}+\frac{Q_{m}^{2}}{\Delta z_{m}^{2}}\right) \delta \Delta z^{2}
\end{aligned}
$$

donde

$$
Q_{p s}=\frac{\Delta z_{m} \Delta T_{p m} k_{p s}}{2 \Delta T_{m} \Delta z_{p w}} \quad Q_{p u}=\frac{\Delta z_{m} \Delta T_{F} k_{F i}}{2 \Delta T_{m} \Delta z_{p i}}
$$

En la Figura 3 se ha representado el error calculado a partir por la ecuación [5] en función de la incertidumbre relativa de cada uno de los parámetros que intervienen en ella. Se observa que los errores cometidos en las medidas de distancia y de temperatura son determinantes en la incertidumbre de la medida de la conductividad. Teniendo en cuenta que el error en la medida de la distancia está determinado por el diámetro de los termopares, por lo que es fijo y en torno al 5\%, para minimizar el error en la conductividad se puso especial cuidado en la medida de temperatura, realizándose una calibración de todos los termopares que permitió dar los valores de $\Delta \mathrm{T}$ en cada patrón con una precisión de $\pm 0,5^{\circ} \mathrm{C}$. Esto aseguraría un error en la determinación del gradiente de temperatura en la columna de medida siempre inferior a \pm $5^{\circ} \mathrm{C}$. Para hacer una estimación del valor de la incertidumbre en la medida de la conductividad se han considerado valores próximos a los reales: $\Delta \mathrm{Z}_{\mathrm{m}}=\Delta \mathrm{Z}_{\mathrm{ps}}=\Delta \mathrm{Z}_{\mathrm{pi}}=20,00 \pm 0,08 \mathrm{~cm}$, $\mathrm{k}_{\mathrm{ps}}=\mathrm{k}_{\mathrm{pi}}=14,00 \pm 0,02 \mathrm{~W} / \mathrm{mK}, \Delta \mathrm{T}_{\mathrm{m}}=\Delta \mathrm{T}_{\mathrm{ps}}=\Delta \mathrm{T}_{\mathrm{pi}}=30,0 \pm 0,5^{\circ} \mathrm{C}$. De esta forma, para un material de conductividad térmica 10

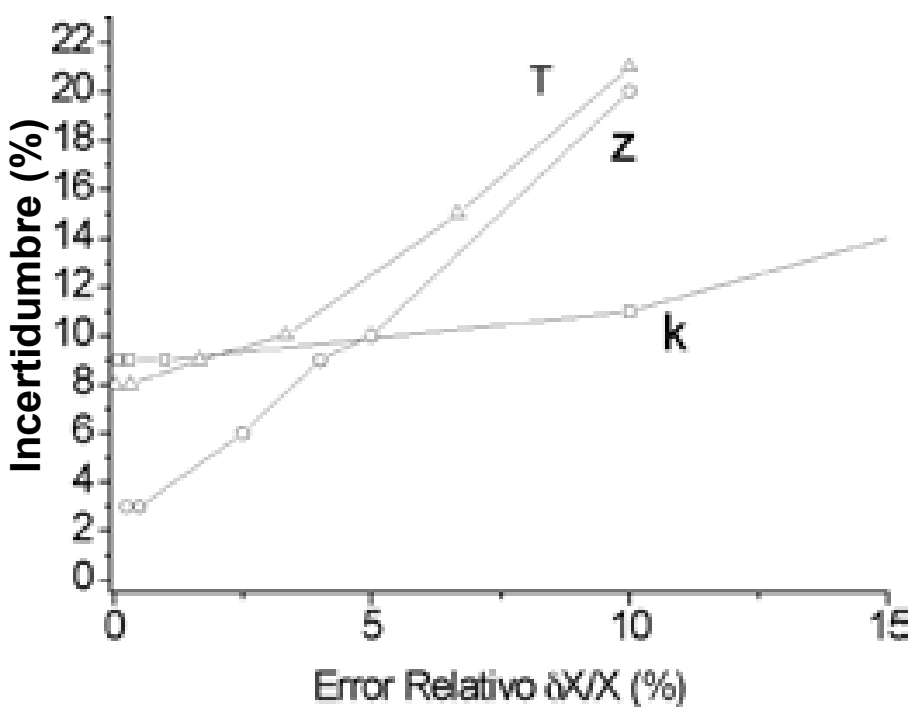

Fig. 3.- Incertidumbre de la medida de conductividad por el método comparativo en función de la incertidumbre de cada uno de los parámetros: temperatura, distancia entre termopares y conductividad de los patrones.

$\mathrm{W} / \mathrm{mK}$, se obtiene una incertidumbre del $9 \%$. Si $\Delta \mathrm{T}$ fuese de $15,0^{\circ} \mathrm{C}$ en cada una de las probetas, la incertidumbre sería del $10 \%$.

\subsection{Parámetros experimentales que afectan al error}

La incertidumbre calculada en el apartado 3.1. está asociada únicamente a las desviaciones causadas por los instrumentos de medida $\mathrm{y}$, por tanto, no tiene en cuenta las posibles desviaciones experimentales respecto a las c.c. asumidas en la resolución de la ecuación del calor, que pueden dar lugar a errores importantes en la determinación de la conductividad.

Asegurar un flujo de calor unidireccional es una de las principales dificultades experimentales en todos los métodos estacionarios. En el caso concreto del método comparativo, la existencia de flujo lateral de calor impide que el flujo de calor a través de los patrones y la muestra sea igual. Para evitar este problema, se emplea un cilindro de guarda alrededor del apilamiento de medida, construido con materiales aislantes y que lleva incorporada una serie de resistencias laterales que se utilizan para producir un gradiente de temperatura similar al alcanzado en el apilamiento. El problema del flujo lateral se complica cuando se mide una muestra de conductividad térmica muy diferente a la de los patrones, ya que es más difícil conseguir un gradiente no lineal en el apilamiento con las resistencias de la guarda.

Cualquier variación en las temperaturas conduce a errores importantes en la medida de los diferentes gradientes y en la conductividad térmica, por lo que debe asegurarse que se han alcanzado las condiciones estacionarias comprobando que la temperatura de todos los termopares del apilamiento no varía durante un periodo de tiempo fijado previamente.

Otra hipótesis asumida en la resolución de la ecuación del calor es que la conductividad térmica es constante a lo 
largo del espesor de las probetas, tanto en los patrones como en el material a ensayar, e igual al valor de la conductividad térmica a la temperatura promedio de cada pieza. Esto equivale a suponer que la conductividad varía linealmente con la temperatura. Sin embargo, esto generalmente no sucede y la conductividad empleada para el cálculo es tanto más diferente de la real cuanto mayor es el gradiente de temperatura empleado. Aunque este error se puede reducir disminuyendo el gradiente térmico, esto hace que el error relativo debido a la incertidumbre en la medida de la temperatura aumente significativamente, por lo que se debe llegar a un compromiso.

Las resistencias térmicas de contacto también influyen de forma importante ya que causan una falta de homogeneidad en la distribución de temperaturas, que provoca una pérdida de flujo hacía el entorno. Para reducir las resistencias de contacto entre las superficies de las probetas, éstas deben tener un perfecto acabado superficial. Además, su efecto se puede reducir utilizando una carga axial o colocando entre las dos superficies una lámina fina flexible de un material con elevada conductividad térmica y alta emisividad $(12,13)$.

Finalmente, las probetas a ensayar deben mecanizarse con precisión y estar perfectamente alineadas con los patrones para que el área a través del cuál circula el flujo de calor sea la igual para las tres probetas.

\section{DISEÑO DEL EQUIPO DE MEDIDA}

El diseño del equipo (Figura 4) se abordó siguiendo las recomendaciones recogidas en la norma ASTM E1225. Para mantener el apilamiento de medida en posición y facilitar el contacto térmico entre las superficies de las muestras, se utilizó un tornillo de apriete. Los elementos calefactores del horno se controlan con dos termopares, uno en el calentador superior $\left(\mathrm{T}_{\mathrm{CS}}\right)$ y otro en el calentador inferior $\left(\mathrm{T}_{\mathrm{CI}}\right)$. Existen, además, otros 6 termopares, correlativos desde arriba hacía abajo, que se utilizan para medir los gradientes de temperatura en cada uno de los patrones y en la muestra. Estos termopares se introducen dentro de una cavidad cilíndrica radial mecanizada en la muestra, de longitud igual al radio de la probeta, y cuya dirección es perpendicular al eje del cilindro para que el termopar esté en un plano isotermo y no haya conducción de calor a través de él (Figura 5). Así, los termopares miden la temperatura en el eje del cilindro, en la parte superior e inferior de cada probeta. Además, los dos termopares de cada una de las probetas están girados $120^{\circ}$ respecto a los de la probeta inmediatamente inferior.

El apilamiento de medida está rodeado por material aislante $\mathrm{y}$ un cilindro de guarda que dispone de dos calentadores laterales, controlados por los termopares $\mathrm{T}_{\mathrm{GS}} \mathrm{y} \mathrm{T}_{\mathrm{GI}}$. El termopar $\mathrm{T}_{\mathrm{GM}}$ tiene como función comprobar el gradiente térmico que se establece en la guarda. De acuerdo con la norma ASTM E1225, se seleccionó una razón entre el radio de la pila de medida y el radio interno del cilindro de guarda de 3 .

Los materiales que se emplearon en las diferentes partes del horno se seleccionaron en función de sus propiedades térmicas y mecánicas, teniendo en cuenta las solicitaciones termomecánicas a las que están sometidos. La selección se realizó a partir de simulaciones numéricas por elementos finitos usando el programa ANSYS (proyecto Cycit-Feder 2FD19970345). Para la zona interna de la guarda (Figura 4), cercana al apilamiento de medida y en contacto con las resistencias, se utilizó fibra aislante (Bulk Fibers, Detrick, R.U.) de $5 \mathrm{~cm}$

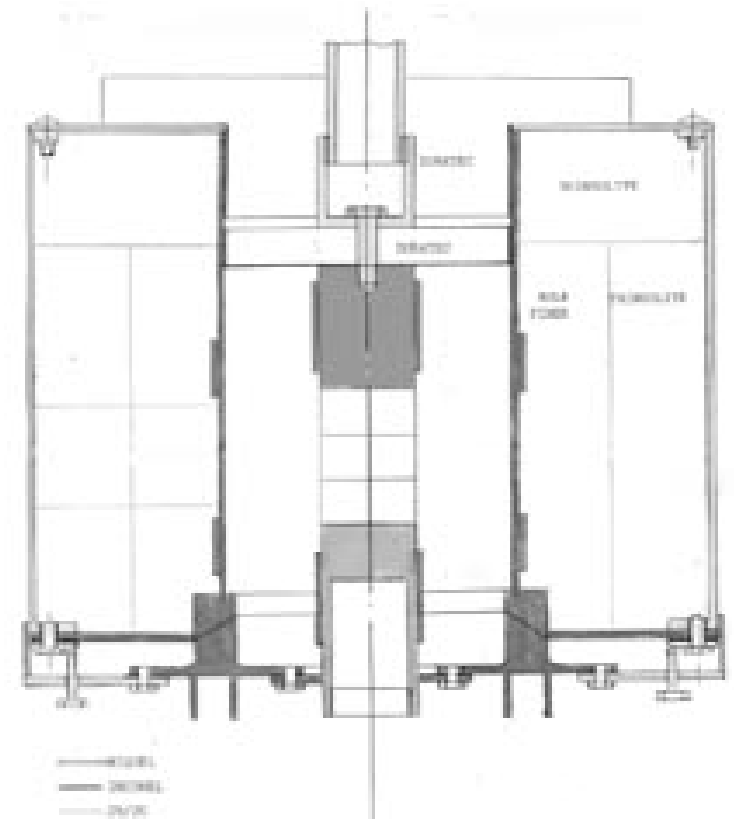

Fig. 4.- Esquema del horno comparativo en el que se muestran los materiales empleados en las distintas partes del horno.

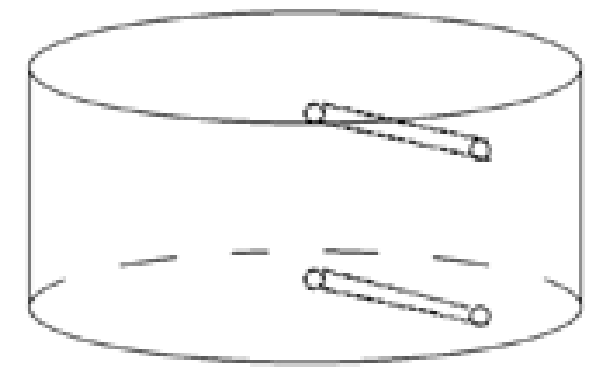

Fig. 5.- Esquema que representa la probeta de ensayo, donde se muestran las dos cavidades cilíndricas, de diámetro igual al del termopar y profundidad igual al radio de la muestra, para alojar a los termopares.

de espesor, con buena estabilidad térmica y conductividad térmica muy baja $\left(0,05 \mathrm{~W} / \mathrm{mK}\right.$ a $200^{\circ} \mathrm{C}$ y $0,22 \mathrm{~W} / \mathrm{mK}$ a $\left.1000^{\circ} \mathrm{C}\right)$. El resto del cilindro de guarda debe soportar temperaturas inferiores por lo que se fabricó con aislante vítreo compacto Thinsulite (Detrick, R.U.), con menor conductividad térmica $\left(0,026 \mathrm{~W} / \mathrm{mK}\right.$ a $200^{\circ} \mathrm{C}$ y $0,046 \mathrm{~W} / \mathrm{mK}$ a $\left.600^{\circ} \mathrm{C}\right)$. La parte exterior de la guarda se fabricó en acero mientras que el cilindro interior es de Inconel. Las resistencias que forman los calentadores superior e inferior están enrolladas alrededor de piezas cilíndricas de níquel, material que posee elevada conductividad térmica $(90 \mathrm{~W} / \mathrm{mK})$. Finalmente, la columna vertical se cierra con un aislante térmico de silicato cálcico $(0,4 \mathrm{~W} / \mathrm{mK})$, con buena resistencia mecánica hasta $1000^{\circ} \mathrm{C}$ (Duratec 1000, Promat GmbH, Alemania). Para evitar perdidas 
de calor por convección, el espacio comprendido entre la pila de medida y la guarda se rellena de un material aislante, consistente en esferas huecas de alúmina de $3,78 \mathrm{~mm}$ de diámetro.

La simulación numérica del horno mostró una insuficiente evacuación de calor por la parte inferior a temperaturas medias y bajas, de forma que no se podía alcanzar el gradiente programado en el apilamiento de medida, por lo que se incorporó un disipador de calor adicional. Este disipador, que se atornilla exteriormente en la parte inferior del horno, alineado con el apilamiento de medida, fue fabricado en aluminio por su alta conductividad térmica, su baja densidad y su alta ductilidad.

Un sistema de control, compuesto por los controladores

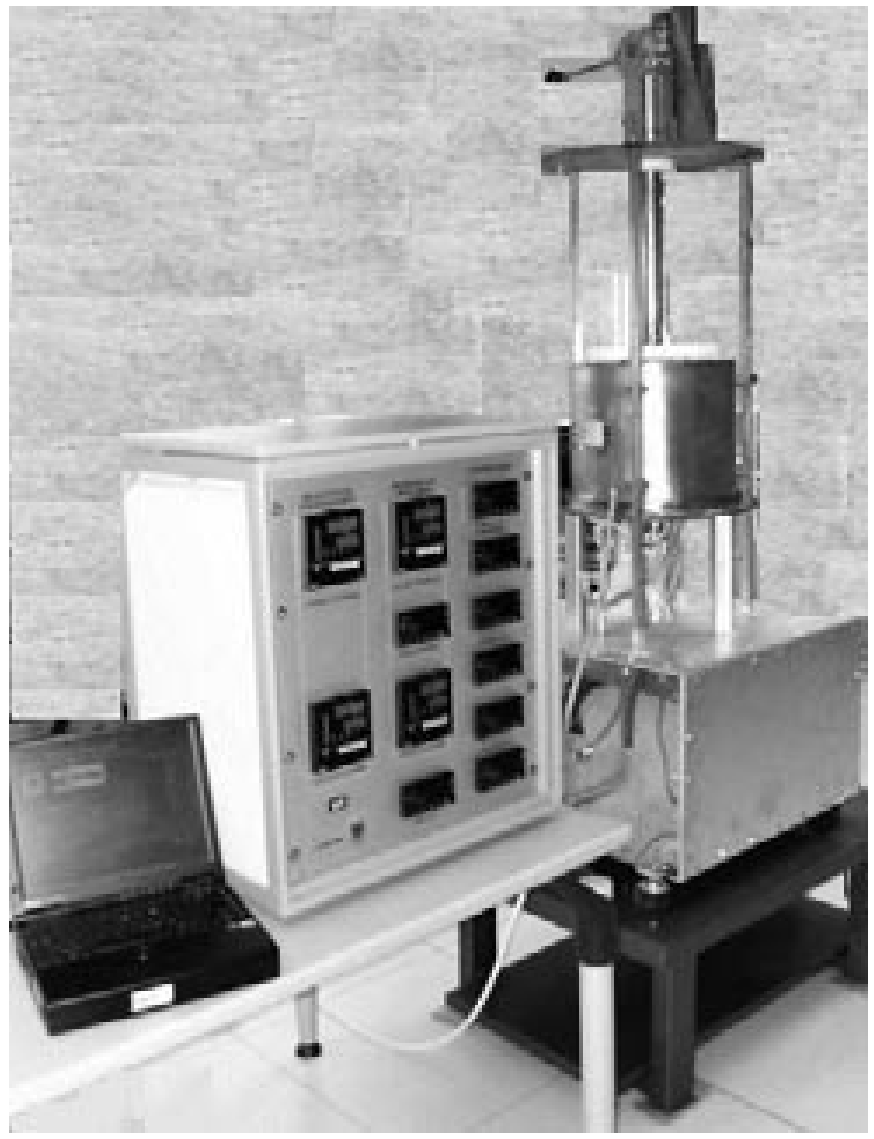

Fig. 6.- Equipo completo desarrollado constituido por el horno, el sistema de control de temperaturas y el programa para el control de los ensayos y la toma de datos.

de las temperaturas de los calentadores principales y del cilindro de guarda y los visualizadores de las temperaturas de las 4 probetas, y un programa para el control de los ensayos, completan el banco de medida desarrollado (Figura 6).

Para realizar las medidas se necesita un conjunto de patrones que cubran el intervalo de conductividades seleccionado, ya que el patrón debe tener conductancia térmica próxima a la del material que se está caracterizando. Existen varios materiales de referencia $(10,14)$ cuya conductividad térmica ha sido avalada mediante pruebas realizadas en distintos laboratorios. En concreto, la norma ASTM E1225 propone el grupo de patrones que se recoge en la Tabla I. En nuestro caso, se seleccionaron los siguientes patrones (en forma de cilindros de $50 \mathrm{~mm}$ de diámetro y $20 \mathrm{~mm}$ de altura, suministrados por
Holometrix, Inc. (EE.UU)): el Fe electrolítico $\left(74 \mathrm{~W} / \mathrm{mK}\right.$ a $\left.25^{\circ} \mathrm{C}\right)$, la aleación Inconel $718\left(11,1 \mathrm{~W} / \mathrm{mK}\right.$ a $\left.25^{\circ} \mathrm{C}\right)$, el Pyroceram 9606 $\left(4,01 \mathrm{~W} / \mathrm{mK}\right.$ a $\left.25^{\circ} \mathrm{C}\right)$ y el Pyrex $7740\left(1,09 \mathrm{~W} / \mathrm{mK}\right.$ a $\left.25^{\circ} \mathrm{C}\right)$, lo que nos permite cubrir el intervalo entre 1 y $100 \mathrm{~W} / \mathrm{mK}$.

TABLA I. MATERIALES PROPUESTOS COMO PATRONES EN LA NORMA ASTM E1225 (10).

\begin{tabular}{|c|c|c|c|}
\hline Material Patrón & Temperatura [K] & $\begin{array}{c}\text { Conductividad } \\
\text { térmica } \\
{[\mathrm{W} / \mathrm{mK}]}\end{array}$ & $\begin{array}{c}\text { Incertidumbre } \\
{[\%]}\end{array}$ \\
\hline $\begin{array}{l}\text { Fe electrolítico } \\
\text { SRM } 734\end{array}$ & Hasta 1000 & $\begin{array}{c}96,71-0,08 \\
\mathrm{~T}+1,8910^{-7} \\
\mathrm{~T}^{2}+1,3010^{-8} \\
\mathrm{~T}^{3}\left(^{*}\right)\end{array}$ & 2 \\
\hline $\begin{array}{c}\text { Acero } \\
\text { Austenítico } \\
\text { SRM } 735\end{array}$ & De 4 a 1200 & $\begin{array}{c}1,22 \mathrm{~T}^{0,432} \mathrm{a} \\
\mathrm{T}>200 \mathrm{~K}\end{array}$ & Menos del 5\% \\
\hline $\begin{array}{l}\text { Wolframio } \\
\text { SRM } 730\end{array}$ & $\begin{array}{c}\text { De } 4 \text { a } 300 \\
300 \text { a } 2000 \\
\text { Más de } 2000\end{array}$ & & $\begin{array}{c}2 \\
2 \text { a } 5 \\
5 \text { a } 8\end{array}$ \\
\hline Hierro & De 80 a 1200 & & 2 \\
\hline Cobre & De 90 a 1250 & $\begin{array}{c}416,3- \\
0,05904 \mathrm{~T}+7,087 \\
10^{7} / \mathrm{T}^{3}\end{array}$ & Menos de 2 \\
\hline Pyroceram 9606 & De 90 a 1200 & $\begin{array}{c}6-0,01 \mathrm{~T}+1,64 \\
10^{-5} \mathrm{~T}^{2}-1,23 \\
10^{-8} \mathrm{~T}^{3} \\
+3,5710^{-12} \mathrm{~T}^{4} \\
\left.(\mathrm{~T}>300 \mathrm{~K}){ }^{*}\right)\end{array}$ & \\
\hline Sílice Fundida & Hasta 1300 & $\begin{array}{c}84,7 / \\
\mathrm{T}+1,48+4,9410^{-} \\
{ }^{4} \mathrm{~T}+9,610^{-13} \mathrm{~T}^{4}\end{array}$ & Menos de 8 \\
\hline Pyrex 7740 & 90 a 600 & $\begin{array}{c}0,16+0,005 \\
\mathrm{~T}-8,22910^{-6} \mathrm{~T}^{2} \\
+5,7710^{-9} \mathrm{~T}^{3}\left(^{*}\right)\end{array}$ & 6 \\
\hline
\end{tabular}

\section{CALIBRACIÓN DEL EQUIPO}

Para la puesta en marcha del equipo, el primer paso fue la calibración de todos los termopares para asegurar un error en la determinación del gradiente de temperatura total en la columna de medida no superior a $\pm 5^{\circ} \mathrm{C}$, para lo que se utilizó un horno portátil (Minical 110-1100C, Societé Pyrox). Para analizar el efecto de diferentes parámetros del ensayo se emplearon los patrones Pyrex y Pyroceram como muestras problema en los siguientes apilamientos: Pyroceram/ Pyrex/Pyroceram y Inconel/Pyroceram/Inconel. En cada experimento se fijaron 5 parámetros: las temperaturas de los calentadores superior e inferior $\left(\mathrm{T}_{\mathrm{CS}}\right.$ y $\left.\mathrm{T}_{\mathrm{CI}}\right)$, que determinan el gradiente en el apilamiento de medida, las temperaturas en la parte superior e inferior de la guarda $\left(\mathrm{T}_{\mathrm{GS}} \mathrm{y} \mathrm{T}_{\mathrm{GI}}\right)$ y el empleo o no del disipador. En cada ensayo se recogieron 11 temperaturas: las temperaturas en los calentadores $\left(\mathrm{T}^{\prime}{ }_{\mathrm{CS}} \mathrm{y}\right.$ $\mathrm{T}_{\mathrm{CI}}^{\prime}$ ) y en la guarda $\left(\mathrm{T}_{\mathrm{GS}}^{\prime} \mathrm{T}^{\prime}{ }_{\mathrm{GM}} \mathrm{y} \mathrm{T}_{\mathrm{GI}}^{\prime}\right)$, que en algunas ocasiones no coincidían con las programadas, y las 6 temperaturas de las tres probetas. El empleo de patrones como muestras permitió comparar la conductividad térmica $\mathrm{K}_{\mathrm{M}^{\prime}}$ deducida de la expresión [4], con el valor recogido en las tablas de datos del patrón a la temperatura media, $\mathrm{K}_{\mathrm{T}}$. De esta forma, el error relativo cometido en cada medida se puede expresar como un porcentaje de $\mathrm{K}_{\mathrm{T}}$. Además, puede calcularse la potencia que circula a través de cada una de las probetas, incluyendo el patrón que hace de muestra, de forma que, 
comparando estas potencias, se obtiene información adicional sobre el experimento, como, por ejemplo, si existen pérdidas o ganancias de calor a lo largo del apilamiento.

En estos ensayos se observó que al aumentar el gradiente de temperatura en el apilamiento, la dificultad para disipar calor en la parte inferior del horno aumentaba. Además, es esperable que $\mathrm{K}_{\mathrm{M}}$ se desvíe de $\mathrm{K}_{\mathrm{T}}$ al aumentar el gradiente debido a que la dependencia de la conductividad térmica con la temperatura generalmente no es lineal. De estos ensayos se dedujo que usando $\mathrm{T}_{\mathrm{CS}}-\mathrm{T}_{\mathrm{CI}} \leq 100^{\circ} \mathrm{C}$ se obtiene un error relativo de $\sim 10 \%$, del mismo orden que la incertidumbre calculada usando la expresión [5].

La influencia del gradiente en la guarda se analizó comparando diferentes ensayos programados con el mismo $\Delta \mathrm{T}$ en el apilamiento de medida, pero con distintos $\Delta \mathrm{T}$ en la guarda. Los resultados mostraban que la temperatura fijada para $\mathrm{T}_{\mathrm{GS}} \mathrm{y}$ el $\Delta \mathrm{T}$ seleccionado en la guarda tenían una fuerte influencia en los resultados del ensayo, observándose que las variaciones de potencia entre las tres probetas podían alcanzar valores superiores al $40 \%$.

También se analizó la influencia de la conductividad térmica del material en el ensayo. Las pérdidas de calor lateral aumentaban al disminuir la conductividad térmica debido a que la diferencia entre las conductividades de los materiales del apilamiento y de la guarda se hacen menores. Este efecto es más significativo a temperaturas altas, de forma que, para un ensayo realizado a $300^{\circ} \mathrm{C}$, se observan pérdidas del $24 \%$ para el patrón Pyrex y ganancias del 12\% para el patrón Pyroceram. La utilización de disipador fue necesaria en todos los ensayos realizados con Pyrex y sólo a bajas temperaturas para materiales de mayor conductividad térmica.

Según Laubitz $(8,15)$, no existe una relación entre la diferencia de potencia entre el patrón superior e inferior $\left(\mathrm{W}_{\mathrm{SI}}\right)$ y el error en la conductividad térmica. Sin embargo, la Figura 7 , en la que se ha representado el error obtenido en $\mathrm{K}_{\mathrm{M}}$ frente al valor absoluto de $\mathrm{W}_{\mathrm{SI}}$ para las muestras patrón, muestra que la incertidumbre de la medida es inferior al 10\% cuando $\left|\mathrm{W}_{\mathrm{sI}}\right| \leq 20 \%$. Por tanto, se escogió éste valor de $\mathrm{W}_{\mathrm{SI}}$ para validar cualquier ensayo.

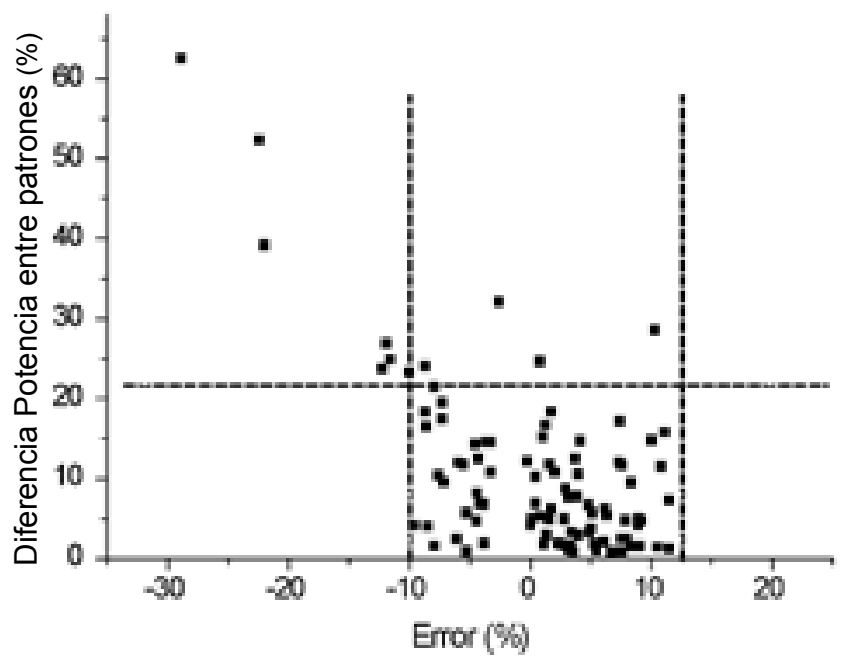

Fig. 7.- Valor absoluto de la diferencia de potencia entre el patrón inferior y el superior en función del error en la medida de la conductividad.
Finalmente, hay que remarcar que los perfiles de temperaturas obtenidos en ensayos repetidos en las mismas condiciones mostraban diferencias de temperatura inferiores a $0,2^{\circ} \mathrm{C}$ para todos los termopares de medida. Estas variaciones daban lugar a diferencias del $2 \%$ en las potencias y desviaciones del $2 \%$ en el error, independientemente de la temperatura de ensayo.

\section{CONDUCTIVIDAD TÉRMICA EN MATERIALES POROSOS}

Los resultados obtenidos con el equipo diseñado han sido validados mediante un análisis comparativo con los valores obtenidos con la técnica del pulso láser, en muestras altamente porosas. Estos ensayos se realizaron a temperaturas comprendidas entre 125 y $600^{\circ} \mathrm{C}$, utilizando un refractario comercial de mullita electrofundida (MU75AT, Norton Ibérica, C.I.S.A.), de densidad 2,5 g. $\mathrm{cm}^{-3}$ y cuya porosidad, calculada asumiendo que su densidad teórica es la de la mullita, es del $21,4 \%$.

Para el equipo comparativo se utilizó una probeta cilíndrica de $50 \mathrm{~mm}$ de diámetro y $15,45 \mathrm{~mm}$ de altura, y el patrón usado fue Pyroceram. En la Figura 8 se observa que la conductividad térmica disminuye de 2 a $1,6 \mathrm{~W} / \mathrm{m} . \mathrm{K}$ en el intervalo de temperaturas analizado.

Con el método del pulso láser se midió la difusividad térmica utilizando discos de $12,7 \mathrm{~mm}$ de diámetro y $1 \mathrm{~mm}$ de espesor. A partir de estos valores, se calculó la conductividad térmica utilizando la expresión: $K=\rho . C_{p}$. $\alpha$, donde $\rho$ es la densidad del refractario y $\mathrm{C}_{\mathrm{p}}$ es el calor específico de la mullita (16). Los valores obtenidos en este caso (Figura 8) son superiores en un $43 \%$ a los obtenidos con el método comparativo. Estas diferencias se acortan considerablemente cuando se corrige el valor obtenido por la técnica del pulso láser considerando la penetración de láser en los poros de la superficie $(5,6)$. De esta forma, al espesor medido con el tornillo micrométrico se le ha restado dos veces el valor de la rugosidad superficial máxima de la muestra $(63 \mu \mathrm{m})$. Los

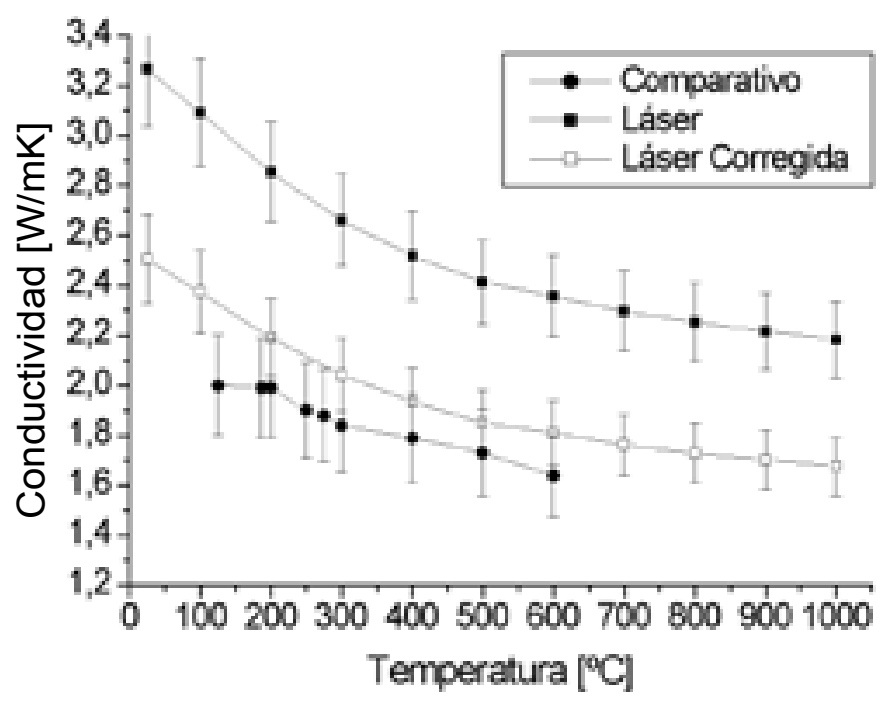

Fig. 8.- Conductividad del refractario MU75AT medida con el método del pulso láser y con el método comparativo, junto con los valores del pulso láser corregidos. 
valores de conductividad térmica corregidos son similares a los medidos con el equipo comparativo, pues están dentro del error de medida de cada uno de los equipos.

\section{CONCLUSIONES}

El equipo comparativo diseñado permite medir la conductividad térmica con una precisión del $10 \%$. El parámetro crítico determinante del error de medida es el gradiente de temperatura, por lo que resulta imprescindible la calibración periódica de los termopares, y se debe asegurar una diferencia de potencias entre el patrón superior y el inferior por debajo del $20 \%$.

La comparación entre las medidas realizadas con este equipo y con el equipo del pulso láser ha permitido validar los resultados obtenidos con el equipo diseñado.

\section{AGRADECIMIENTOS}

Este trabajo ha sido financiado por los proyectos CAM 07N/ 0095/ 2002 y Mcyt, MAT 2003-06147-C04-01.

Se agradece a la empresa Nortón Ibérica C.I.S.A. el material refractario suministrado.

\section{BIBLIOGRAFÍA}

1. K.C. Mills, "Review of methods for the measurement of thermal conductivities of sand-, investment- and die-mold materials", Natl. Phys. Lab. (U. K.), [Rep.], CMMT(A) 110 62-77 (1998).
2. G.S. Sheffield, J.R. Schorr, "Comparison of thermal diffusivity and thermal conductivity methods", Ceramic Bull. 1 [70] 102-106 (1991).

3. E. Garcia, R. Martínez, M.I. Osendi, P. Miranzo, "Método del Pulso láser para la medida de la difusividad térmica en materiales cerámicos." Bol. Soc. Esp. Cerám. V. 40 [4] 289-294 (2001).

4. M. Golombok, L. C. Shirvill "Laser flash thermal conductivity studies of porous metal fiber materials" J. Appl. Phys. 6 [63] 1971-1976 (1988).

5. E. Garcia, M. I. Osendi, P. Miranzo "Thermal diffusivity of porous cordierite ceramic burners" J. Appl. Phys. 5 [92] 2346-2349 (2002).

6. R. Barea, E. Garcia, M.I. Osendi, P. Miranzo, “Thermal Diffusivity of Porous Ceramics" Key Eng. Mat. [264-268] 2179-2182 (2004).

7. J. Francl, W. D. Kingery, "Thermal Conductivity: IV Apparatus for determining thermal conductivity by a comparative method", J. Am. Ceram. Soc. 37 [2] 80-84 (1954).

8. M. J. Laubitz "On measurement of thermal conductivity at high temperatures" Can. J. Phys. [39] 1029-39 (1961)

9. R. P. Tye, "The measurement of thermal conductivity by the comparative method", Cap. 3, en Thermal Conductivity Vol 2, Ed. Academic Press, London (1969).

10. Norma ASTM E1225, "Test Method for thermal conductivity of solid bu means of the guarded comparative longitudinal heat-flow techniques" ASTM Stand., Filadelfia, (1987).

11. C. Sánchez del Río. "Análisis de Errores", Ed. Eudema Universitario (1989).

12. V. V. Mirkovich, "Comparative Method and Choice of Standards for thermal conductivity determinations", J. Am. Ceram. Soc. 8 [48] 387-391 (1965).

13. C. G. S Pillai, A. M George, "An Improved comparative thermal conductivity apparatus for measurements at high temperatures", Int. J. Thermophys. 3 [12] 563-76 (1991)

14. L. Filoni, G. Rocchini, "Thermal conductivity of iron, plain carbon and stainless steels, and Inconel 718 from $360 \mathrm{~K}$ to 900 K" , High Temp.- High Pressures 19 [4] 381-387 (1987).

15. M. J. Laubitz, "Mathematical Treatment of a four component furnace", Can J. Phys. [39] 951-955 (1961)

16. M.W. Chase, NIST-JANAF Thermochemical Tables, Monograph 9, 4th Edition, Ed. Journal of Physical and Chemical Reference Data (1996).

Recibido: 15.01 .05

Aceptado: 23.12.05 